\title{
On the Evolutionary Dynamics of the Cahn-Hilliard Equation with Cut-Off Mass Source
}

Chaeyoung Lee, Hyundong Kim, Sungha Yoon, Jintae Park, Sangkwon Kim, Junxiang Yang and Junseok Kim*

Department of Mathematics, Korea University, Seoul 02841, Republic of Korea

Received 31 March 2020; Accepted (in revised version) 12 July 2020

\begin{abstract}
We investigate the effect of cut-off logistic source on evolutionary dynamics of a generalized Cahn-Hilliard (CH) equation in this paper. It is a well-known fact that the maximum principle does not hold for the $\mathrm{CH}$ equation. Therefore, a generalized $\mathrm{CH}$ equation with logistic source may cause the negative concentration blow-up problem in finite time. To overcome this drawback, we propose the cut-off logistic source such that only the positive value greater than a given critical concentration can grow. We consider the temporal profiles of numerical results in the one-, two-, and three-dimensional spaces to examine the effect of extra mass source. Numerical solutions are obtained using a finite difference multigrid solver. Moreover, we perform numerical tests for tumor growth simulation, which is a typical application of generalized $\mathrm{CH}$ equations in biology. We apply the proposed cut-off logistic source term and have good results.
\end{abstract}

AMS subject classifications: 65M06, 65M55, 65Z05, 68U20

Key words: Cahn-Hilliard equation, logistic source, finite difference method, tumor growth application.

\section{Introduction}

In this paper we investigate numerically the evolutionary dynamics of the following generalized Cahn-Hilliard (CH) equation with nonlinear source $\gamma(\phi(\mathbf{x}, t))$ and further examine the effect of cut-off source $\gamma_{c}(\phi(\mathbf{x}, t))$,

$$
\begin{aligned}
& \frac{\partial \phi(\mathbf{x}, t)}{\partial t}=\Delta \mu(\mathbf{x}, t)+\gamma(\phi(\mathbf{x}, t)), \quad \mathbf{x} \in \Omega, \quad t>0, \\
& \mu(\mathbf{x}, t)=F^{\prime}(\phi(\mathbf{x}, t))-\epsilon^{2} \Delta \phi(\mathbf{x}, t), \\
& \mathbf{n} \cdot \nabla \phi(\mathbf{x}, t)=\mathbf{n} \cdot \nabla \mu(\mathbf{x}, t)=0, \quad \mathbf{x} \in \partial \Omega,
\end{aligned}
$$

${ }^{*}$ Corresponding author. Email address: cfdkim@korea.ac.kr (J. Kim) 
where $\phi(\mathbf{x}, t)(0 \leq \phi \leq 1)$ is the order parameter of phase-field model, which represents the concentration of one species in the binary mixtures in the domain $\Omega \subset \mathbb{R}^{d}(d=$ $1,2,3)$. The variational derivative $F^{\prime}(\phi)=\phi^{3}-1.5 \phi^{2}+0.5 \phi$ is derived from $F(\phi)=$ $0.25 \phi^{2}(\phi-1)^{2}$, which is a double-well potential, a positive constant $\epsilon$ is related to the thickness of interfacial transition, and $\mathbf{n}$ is the outward normal vector at the domain boundary. Note that Eqs. (1.1) and (1.2) without $\gamma(\phi)$ are the $\mathrm{CH}$ equation, which is originally devised to represent the coarsening dynamics and phase separations in binary alloy [5]. We refer the readers to a review paper [21] and the references therein for more detailed physical, mathematical, and numerical derivations for the $\mathrm{CH}$ equation. The $\mathrm{CH}$ type models are applied in various fields such that bacterial films [20], thin films [28], image inpainting [3], population dynamics [7], etc. The $\mathrm{CH}$ equation with Eq. (1.3) yields the conservation of mass of $\phi$, the existence of global attractors in finite dimensional space has been proved as the follow-up research [27]. In addition, the equilibrium solutions of the $\mathrm{CH}$ equation were studied especially on the effect of the dimensions of space [23].

In slightly different sense, the generalized model, Eqs. (1.1) and (1.2), has been employed throughout biological entities, for instance, the growth simulation of tumors. Typically in tumor growth research, appropriate mathematical models and computational simulations have important roles on diagnosis and treatment, along with clinical and experimental data [29]. Since the interactions between cellular proliferation, adhesion, and other properties are all concerned with tissue growth, it is important to simulate diffuse-interface kinetics in which tissue growth occurs. Therefore, a lot of studies related on tumor growth modeling have been conducted. Huang et al. [13] addressed numerical computations of biological models on unbounded domains. Efficient local absorbing boundary conditions and linearized finite difference method were built and presented to solve the reduced problem. The authors in $[11,34]$ developed a thermodynamically consistent diffuse-interface model of multispecies tumor growth with complex morphologies by using the $\mathrm{CH}$ type model with substrate components. They presented the proof of thermodynamic consistency of the multispecies mixture model and provided numerical simulations of unstable avascular tumor growth and malignant development such as tumor invasion, tumor-induced angiogenesis in both twoand three-dimensional spaces. Another related study of thermodynamic consistency is a four-species tumor growth model consisting of both tumor and healthy cells and extracellular species, which is proposed by the authors in [12]. They presented various numerical simulation results with several model parameters, and confirmed that the profit of usage of diffuse-interface models in order to incorporate multiphase in a single model. Jiang et al. [16] described a phase-field based diffuse-interface model for cell growth, division, and packing of multi-cell aggregation using finite element method. Vilanova et al. [33] presented a model of tumor angiogenesis for vascular regression and regrowth using the Allen-Cahn type equation which is widely used in diffuse-interface modeling. They controlled the growth and regression with the chemical free energy. On the one hand, a level-set based method was used to capture the geometric influences of curvature on the evolution of cell density and tissue shape and 
model tissue growth [1]. Furthermore, several numerical methods are proposed to solve the corresponding mathematical models in $[8,25,35]$, we refer to the readers check these papers and the references therein for more details.

The multiphase growth models listed above are highly complex systems. In particular, designing the mechanism of growth and death of tissue as a source term in model is important; hence it is worth to be investigated. Khain and Sander [17] proposed the generalized $\mathrm{CH}$ model with logistic proliferation. They analyzed both subcritical and supercritical adhesion regions in model. The former has similar propagations in fronts those of the Fisher-Kolomogorov equation, which fit well with the numerical solution of proposed model. The latter has the nontrivial transient action caused by the relaxation process of the $\mathrm{CH}$ equation. As a follow-up study to the same model, Cherfils et al. [6] investigated the asymptotic behavior of the model with Neumann boundary condition. They proved theoretically and numerically that the solution behavior is either a global in time if the density of cells is locally bounded or a blow-up in finite time. Aristotelous et al. [2] presented adaptive, second-order in time discontinuous Galerkin methods for the $\mathrm{CH}$ equation with a nonlinear reaction mass source term. They found good agreements in convergence and robustness of proposed scheme both theoretical and numerical ways. Especially, they adopted the appropriate reaction source term for tumor growth which consists of logistic growth, linear death, and artificial growth terms. Further asymptotic dynamics research with four kinds of mass source, linear, logistic, reaction source in [2], and singular nonlinear source, was conducted by Fakih [10].

Whence we investigate the evolutionary dynamics of $\mathrm{CH}$ equation with a source, we especially focus on extra nonlinear source $\gamma(\phi)$ as a combination of both logistic growth and linear death terms. Cohen and Murray [7] considered a reaction-diffusion model for growth and dispersal in population and investigated spatial effects involving logistic kinetics. They found spatial structures which are stable and unstable. It is possible that an asymptotic behavior of logistic differential equations becomes unstable. Moreover, it is well known that the $\mathrm{CH}$ equation does not satisfy the maximum principle [30]. Therefore, the solution of Eqs. (1.1)-(1.3) can be blown up. To overcome this drawback, we apply the cut-off method [24] to the $\mathrm{CH}$ equation with the logistic source term and perform numerical tests for tumor growth in two- and three-dimensional spaces.

This paper is organized as follows. In Section 2, we describe the numerical solution algorithms of the $\mathrm{CH}$ equation with a source term. In Section 3, we give an explanation of the logistic growth in symmetric and Cartesian coordinates. Then, we propose the cut-off logistic growth term and present the tumor growth simulations to verify the effect of cut-off logistic growth term. Finally, conclusions are drawn in Section 4.

\section{Numerical solution}

In this section, we describe the numerical discretizations for the $\mathrm{CH}$ equation with a source term in symmetric coordinates and Cartesian coordinates. It is simple and fast to investigate the evolution of solution profiles in the symmetric coordinates. In 
addition, when the solutions are nonsymmetric, we consider the evolution in Cartesian coordinates.

\subsection{Symmetric coordinates}

To study the effect of the source term, we fist want to obtain simple solution for the $\mathrm{CH}$ equation. Let $d$ be the space dimension. We present numerical solutions of the $\mathrm{CH}$ equation in symmetric $(d=1)$, radially symmetric $(d=2)$, and spherically symmetric $(d=3)$ coordinates $[14,15]$ :

$$
\begin{aligned}
& \phi_{t}(r, t)=\frac{1}{r^{d-1}}\left[r^{d-1} \mu_{r}(r, t)\right]_{r}+\gamma(\phi(r, t)), \quad r \in \Omega, \quad t>0, \\
& \mu(r, t)=F^{\prime}(\phi(r, t))-\frac{\epsilon^{2}}{r^{d-1}}\left[r^{d-1} \phi_{r}(r, t)\right]_{r} .
\end{aligned}
$$

For simplicity of exposition, let the computational domain be $\Omega=(a, b)$. Let $\Omega_{h}=\left\{r_{i}\right.$ : $\left.r_{i}=a+(i-0.5) h, 1 \leq i \leq N_{r}\right\}$ be the discrete domain, where $N_{r}$ is an even integer and $h=\frac{b-a}{N_{r}}$. Let $\phi_{i}^{n}$ and $\mu_{i}^{n}$ be the numerical approximations of $\phi\left(r_{i}, t^{n}\right)$ and $\mu\left(r_{i}, t^{n}\right)$, respectively, where $t^{n}=n \Delta t$ and $\Delta t$ is the temporal step size. In [14], the authors used the explicit Euler's scheme. On the other hand, we adopt an auxiliary variable $q=\phi(\phi-1)$ and the second-order backward difference formula (BDF2) for temporal discretization [36] to achieve second-order temporal accuracy. Then,

$$
\begin{aligned}
\phi_{t} & =\frac{1}{r^{d-1}}\left[r^{d-1} \mu_{r}(r, t)\right]_{r}+\gamma(\phi(r, t)), \\
\mu & =q(\phi-0.5)-\frac{\epsilon^{2}}{r^{d-1}}\left[r^{d-1} \phi_{r}(r, t)\right]_{r}, \\
q_{t} & =(2 \phi-1) \phi_{t} .
\end{aligned}
$$

The discretization of Eqs. (2.3)-(2.5) is derived as follows:

$$
\begin{aligned}
& \frac{1}{2 \Delta t}\left(3 \phi_{i}^{n+1}-4 \phi_{i}^{n}+\phi_{i}^{n-1}\right)=\Delta_{h} \mu_{i}^{n+1}+\gamma\left(\widetilde{\phi}_{i}\right), \\
& \mu_{i}^{n+1}=q_{i}^{n+1}\left(\widetilde{\phi}_{i}-0.5\right)-\epsilon^{2} \Delta_{h} \phi_{i}^{n+1}, \\
& \frac{1}{2 \Delta t}\left(3 q_{i}^{n+1}-4 q_{i}^{n}+q_{i}^{n-1}\right)=\frac{1}{2 \Delta t}\left(2 \widetilde{\phi}_{i}-1\right)\left(3 \phi_{i}^{n+1}-4 \phi_{i}^{n}+\phi_{i}^{n-1}\right),
\end{aligned}
$$

where $\widetilde{\phi}_{i}=2 \phi_{i}^{n}-\phi_{i}^{n-1}$, the discrete Laplacian is

$$
\begin{aligned}
& \Delta_{h} \phi_{i}^{n+1}=\frac{1}{\left(r_{i}^{d-1} h^{2}\right)}\left[r_{i+\frac{1}{2}}^{d-1}\left(\phi_{i+1}^{n+1}-\phi_{i}^{n+1}\right)-r_{i-\frac{1}{2}}^{d-1}\left(\phi_{i}^{n+1}-\phi_{i-1}^{n+1}\right)\right], \\
& r_{i+\frac{1}{2}}^{d-1}=\frac{1}{2}\left(r_{i}^{d-1}+r_{i+1}^{d-1}\right),
\end{aligned}
$$

and the initial settings are $\phi_{i}^{-1}=\phi_{i}^{0}$ and $q_{i}^{-1}=q_{i}^{0}$. We use the homogeneous Neumann boundary conditions for both $\phi$ and $\mu$. That is,

$$
\phi_{0}^{n+1}=\phi_{1}^{n+1}, \quad \phi_{N_{r}+1}^{n+1}=\phi_{N_{r}}^{n+1}, \quad \mu_{0}^{n+1}=\mu_{1}^{n+1}, \quad \mu_{N_{r}+1}^{n+1}=\mu_{N_{r}}^{n+1} .
$$


The discrete equations (2.6) and (2.7) are solved by using a nonlinear multigrid method [32]. In this study, we focus on the effect of a source term on the evolutionary dynamics of the $\mathrm{CH}$ equation with a source term. Therefore, we skip the numerical solution algorithm. For more details on the full description of the nonlinear multigrid method for the $\mathrm{CH}$ equation, refer to [18].

\subsection{Cartesian coordinates}

For tumor growth simulations, we discretize the governing equations in two- and three-dimensional Cartesian domain if the evolutionary dynamics are not symmetric. First, in two-dimensional domain $\Omega=(a, b) \times(c, d)$, we denote the uniform mesh size as

$$
h=\frac{b-a}{N_{x}}=\frac{d-c}{N_{y}},
$$

where $N_{x}$ and $N_{y}$ are positive even integers, and the computational domain as

$$
\Omega_{h}=\left\{\left(x_{i}, y_{j}\right): x_{i}=a+(i-0.5) h, y_{j}=c+(j-0.5) h, 1 \leq i \leq N_{x}, 1 \leq j \leq N_{y}\right\} .
$$

Let $\phi_{i j}^{n}$ and $\mu_{i j}^{n}$ be the numerical approximations of $\phi\left(x_{i}, y_{j}, t^{n}\right)$ and $\mu\left(x_{i}, y_{j}, t^{n}\right)$, respectively. Here, $\Delta t$ is the temporal step size and $t^{n}=n \Delta t$. We discretize Eqs. (1.1) and (1.2) using the auxiliary variable $q$ and the BDF2 for temporal discretization:

$$
\begin{aligned}
& \frac{1}{2 \Delta t}\left(3 \phi_{i j}^{n+1}-4 \phi_{i j}^{n}+\phi_{i j}^{n-1}\right)=\Delta_{h} \mu_{i j}^{n+1}+\gamma\left(\widetilde{\phi}_{i j}\right), \\
& \mu_{i j}^{n+1}=q_{i j}^{n+1}\left(\widetilde{\phi}_{i j}-0.5\right)-\epsilon^{2} \Delta_{h} \phi_{i j}^{n+1}, \\
& \frac{1}{2 \Delta t}\left(3 q_{i j}^{n+1}-4 q_{i j}^{n}+q_{i j}^{n-1}\right)=\frac{1}{2 \Delta t}\left(2 \widetilde{\phi}_{i j}-1\right)\left(3 \phi_{i j}^{n+1}-4 \phi_{i j}^{n}+\phi_{i j}^{n-1}\right),
\end{aligned}
$$

where $\widetilde{\phi}_{i j}=2 \phi_{i j}^{n}-\phi_{i j}^{n-1}$, the discrete Laplacian is

$$
\Delta_{h} \phi_{i j}^{n+1}=\frac{1}{h^{2}}\left(\phi_{i-1, j}^{n+1}+\phi_{i+1, j}^{n+1}+\phi_{i, j-1}^{n+1}+\phi_{i, j+1}^{n+1}-4 \phi_{i j}^{n+1}\right)
$$

and the initial settings are $\phi_{i j}^{-1}=\phi_{i j}^{0}$ and $q_{i j}^{-1}=q_{i j}^{0}$. We use the homogeneous Neumann boundary conditions (1.3) for both $\phi$ and $\mu$. That is,

$$
\begin{array}{cccc}
\phi_{0 j}^{n+1}=\phi_{1 j}^{n+1}, & \phi_{N_{x}+1, j}^{n+1}=\phi_{N_{x}, j}^{n+1}, & \mu_{0 j}^{n+1}=\mu_{1 j}^{n+1}, & \mu_{N_{x}+1, j}^{n+1}=\mu_{N_{x}, j}^{n+1}, \\
\phi_{i 0}^{n+1}=\phi_{i 1}^{n+1}, & \phi_{i, N_{y}+1}^{n+1}=\phi_{i, N_{y}}^{n+1}, \quad \mu_{i 0}^{n+1}=\mu_{i 1}^{n+1}, & \mu_{i, N_{y}+1}^{n+1}=\mu_{i, N_{y}}^{n+1}
\end{array}
$$

Next, in three-dimensional domain $\Omega=(a, b) \times(c, d) \times(e, f)$, the uniform mesh size is

$$
h=\frac{b-a}{N_{x}}=\frac{d-c}{N_{y}}=\frac{f-e}{N_{z}}
$$


where $N_{x}, N_{y}$, and $N_{z}$ are positive even integers. Then, the discrete domain is

$$
\begin{array}{r}
\Omega_{h}=\left\{\left(x_{i}, y_{j}, z_{k}\right): x_{i}=a+(i-0.5) h, y_{j}=c+(j-0.5) h, z_{k}=e+(k-0.5) h,\right. \\
\left.1 \leq i \leq N_{x}, 1 \leq j \leq N_{y}, 1 \leq k \leq N_{z}\right\} .
\end{array}
$$

Let $\phi_{i j k}^{n}$ and $\mu_{i j k}^{n}$ be the numerical approximations of $\phi\left(x_{i}, y_{j}, z_{k}, t^{n}\right)$ and $\mu\left(x_{i}, y_{j}, z_{k}, t^{n}\right)$, respectively. Here, $\Delta t$ is the temporal step size and $t^{n}=n \Delta t$. Now, we discretize Eqs. (1.1) and (1.2) by using the auxiliary variable $q$ and the BDF2 for temporal discretization:

$$
\begin{aligned}
& \frac{1}{2 \Delta t}\left(3 \phi_{i j k}^{n+1}-4 \phi_{i j k}^{n}+\phi_{i j k}^{n-1}\right)=\Delta_{h} \mu_{i j k}^{n+1}+\gamma\left(\widetilde{\phi}_{i j k}\right), \\
& \mu_{i j k}^{n+1}=q_{i j k}^{n+1}\left(\widetilde{\phi}_{i j k}-0.5\right)-\epsilon^{2} \Delta_{h} \phi_{i j k}^{n+1}, \\
& \frac{1}{2 \Delta t}\left(3 q_{i j k}^{n+1}-4 q_{i j k}^{n}+q_{i j k}^{n-1}\right)=\frac{1}{2 \Delta t}\left(2 \widetilde{\phi}_{i j k}-1\right)\left(3 \phi_{i j k}^{n+1}-4 \phi_{i j k}^{n}+\phi_{i j k}^{n-1}\right),
\end{aligned}
$$

where $\widetilde{\phi}_{i j k}=2 \phi_{i j k}^{n}-\phi_{i j k}^{n-1}$,

$$
\Delta_{h} \phi_{i j k}^{n+1}=\frac{1}{h^{2}}\left(\phi_{i-1, j, k}^{n+1}+\phi_{i+1, j, k}^{n+1}+\phi_{i, j-1, k}^{n+1}+\phi_{i, j+1, k}^{n+1}+\phi_{i, j, k+1}^{n+1}+\phi_{i, j, k-1}^{n+1}-6 \phi_{i j k}^{n+1}\right),
$$

and $\phi_{i j k}^{-1}=\phi_{i j k}^{0}$ and $q_{i j k}^{-1}=q_{i j k}^{0}$. We use the homogeneous Neumann boundary conditions (1.3) for both $\phi$ and $\mu$. That is,

$$
\begin{array}{llll}
\phi_{0 j k}^{n+1}=\phi_{1 j k}^{n+1}, & \phi_{N_{x}+1, j k}^{n+1}=\phi_{N_{x}, j k}^{n+1}, & \mu_{0 j k}^{n+1}=\mu_{1 j k}^{n+1}, & \mu_{N_{x}+1, j k}^{n+1}=\mu_{N_{x}, j k}^{n+1}, \\
\phi_{i 0 k}^{n+1}=\phi_{i 1 k}^{n+1}, & \phi_{i, N_{y}+1, k}^{n+1}=\phi_{i, N_{y}, k}^{n+1}, & \mu_{i 0 k}^{n+1}=\mu_{i 1 k}^{n+1}, & \mu_{i, N_{y}+1, k}^{n+1}=\mu_{i, N_{y}, k}^{n+1}, \\
\phi_{i j 0}^{n+1}=\phi_{i j 1}^{n+1}, & \phi_{i j, N_{z}+1}^{n+1}=\phi_{i j, N_{z}}^{n+1}, & \mu_{i j 0}^{n+1}=\mu_{i j 1}^{n+1}, & \mu_{i j, N_{z}+1}^{n+1}=\mu_{i j, N_{z}}^{n+1} .
\end{array}
$$

We also solve the discrete equations (2.10)-(2.12) and (2.14)-(2.16) using a nonlinear multigrid method $[18,32]$.

\section{Numerical results}

Our goal is to observe the dynamics of the $\mathrm{CH}$ equation with a source term, which consists of a logistic growth term and a death term. First, we consider the effect of logistic growth term and present the cut-off method to eliminate the instabilities of logistic term in the benchmark configuration. Then, we simulate tumor growth with both growth and death terms in two- and three-dimensional space.

In this section, we use $\epsilon_{m}=\frac{m h}{\left[4 \sqrt{2} \tanh ^{-1}(0.9)\right]}$ [19] unless otherwise stated in the numerical tests. Let us briefly explain how to derive this expression. From our choice of the equilibrium profile $\phi(r)=0.5\left(1-\tanh \left(\frac{r}{2 \sqrt{2} \epsilon}\right)\right)$ on an infinite domain, the concentration field varies from 0.05 to 0.95 over a distance of about $\xi=4 \sqrt{2} \epsilon \tanh ^{-1}(0.9)$ as shown in Fig. 1. Therefore, if we want this value to be about $m$ grid points, then we can define $\epsilon_{m}$ by $\frac{m h}{\left[4 \sqrt{2} \tanh ^{-1}(0.9)\right]}$. 


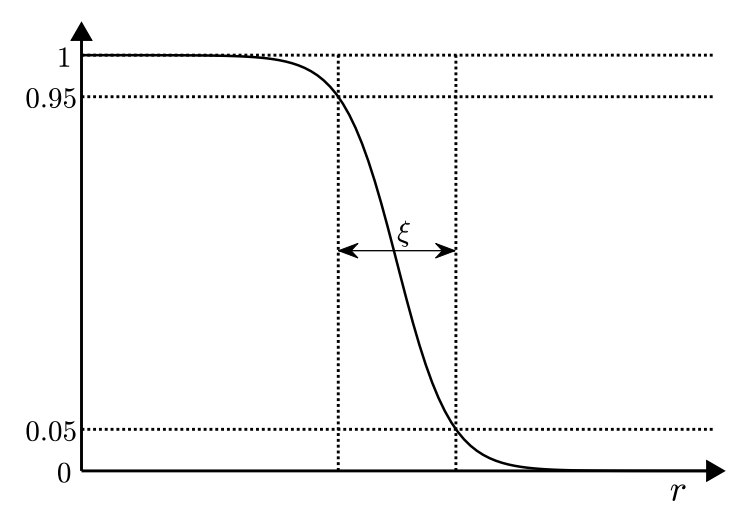

Figure 1: Concentration field varies form 0.05 to 0.95 over a distance of about $\xi=4 \sqrt{2} \epsilon \tanh ^{-1}(0.9)$.

\subsection{Logistic growth}

Before investigating the dynamics of the $\mathrm{CH}$ equation with a logistic source term, we consider the following logistic equation:

$$
\frac{d \phi}{d t}=\gamma_{0} \phi(1-\phi)
$$

where $\gamma_{0}$ is the rate of proliferation [4,26]. The solution of Eq. (3.1) is given as

$$
\phi(t)=\frac{\phi(0)}{\phi(0)+[1-\phi(0)] e^{-\gamma_{0} t}} .
$$

Fig. 2 shows the temporal evolutions of $\phi$ with different initial values $\phi(0)=$ $1.2,0.8,0.05,-0.002$. Here, $\gamma_{0}=1$ is used for the convenience of explanation. If $\phi(0)>0$, then all the solutions approach one as time increases. If $\phi(0)=0$, then we have $\phi(t)=0$ for all time. If $\phi(0)<0$, then the solution decreases as time increases and exhibits a finite-time blowup at $t=\ln [1-1 / \phi(0)] \approx 6.2166$ with $\phi(0)=-0.002$.

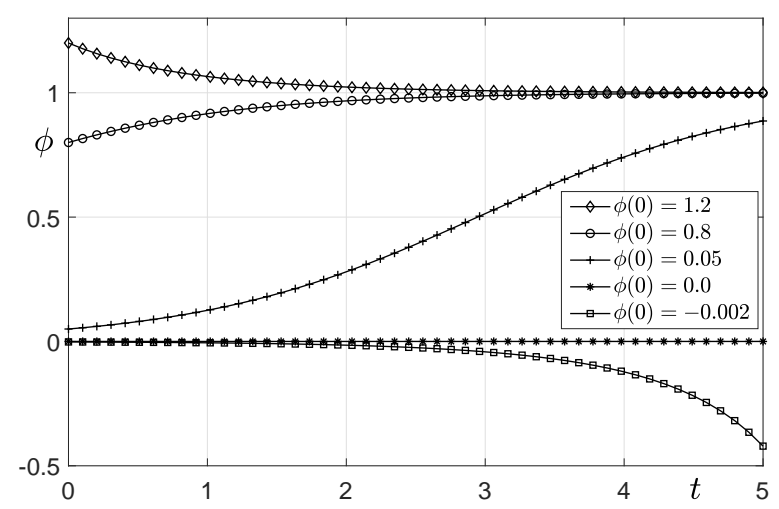

Figure 2: Temporal evolutions of $\phi$ versus $t$ with $\phi(0)=1.2,0.8,0.05,0,-0.002$. 
Next, let us consider the following one-dimensional $\mathrm{CH}$ equation with the logistic growth source term:

$$
\begin{aligned}
& \phi_{t}(r, t)=\mu_{r r}(r, t)+\gamma_{0} \phi(r, t)(1-\phi(r, t)), \quad r \in \Omega=(0,4), \quad t>0, \\
& \mu(r, t)=F^{\prime}(\phi(r, t))-\epsilon^{2} \phi_{r r}(r, t) .
\end{aligned}
$$

To study the effect of logistic growth source term $\gamma(\phi(r, t))$ on the dynamics, we consider an initial condition:

$$
\phi(r, 0)= \begin{cases}1.2, & \text { if } 0 \leq r<1, \\ 0.8, & \text { if } 1 \leq r<2, \\ 0.2, & \text { if } 2 \leq r<3 \\ -0.2, & \text { otherwise }\end{cases}
$$

Fig. 3 shows the computational results with $\gamma_{0}=0$ and $\gamma_{0}=5$ at $t=50 \Delta t$. The simulation parameters used are $N_{r}=256, h=\frac{4}{N_{r}}, \Delta t=10 h^{2}$, and $\epsilon=\epsilon_{16}$. Without the source term (i.e., $\gamma_{0}=0$ ), the $\mathrm{CH}$ dynamics results in phase separation into zero or one. However, with the logistic source term and a nonzero value of $\gamma_{0}$, the logistic dynamics dominates the system. Initially positive or negative phase-field value approaches one or continuously decreases, respectively, as shown in Fig. 2, because it is well known that the $\mathrm{CH}$ equation does not satisfy the maximum principle [30].

In other words, the solution for the $\mathrm{CH}$ equation can be negative and the logistic growth term makes the negative concentration be larger negative value. Eventually it is possible that the solution blows up in finite time. According to the portion of negative $\phi$ of the initial condition, the computational results are completely different with the same $\gamma_{0}$. The initial conditions are defined as follows:

$$
\begin{aligned}
& \text { Fig. } 4(\mathrm{a}): \phi(r, 0)= \begin{cases}1, & \text { if } 0 \leq r<1.5, \\
-0.1, & \text { otherwise },\end{cases} \\
& \text { Fig. } 4(\mathrm{~b}): \phi(r, 0)= \begin{cases}1, & \text { if } 0 \leq r<3.5, \\
-0.1, & \text { otherwise, }\end{cases} \\
& \text { Fig. } 4(\mathrm{c}): \phi(r, 0)= \begin{cases}1, & \text { if } 0 \leq r<1.5, \\
0.1, & \text { otherwise. }\end{cases}
\end{aligned}
$$

We set $\gamma_{0}=5$, and the other parameters as the same as used above. Fig. 4 illustrates the different phenomenon depending on the initial settings. When we set relatively large portion of negative $\phi$ as an initial condition, the computational results show the blowup as shown in Fig. 4(a). Meanwhile, $\phi$ increases to one without blow-up as shown in Fig. 4(b) when we use small portion of negative $\phi$. In addition, a phenomenon such as spurious growth occurs as shown in Fig. 4(c). In this paper, these two phenomena are called unstable. Therefore, to overcome these drawbacks, we apply the cut-off method [24] to the logistic source term. 


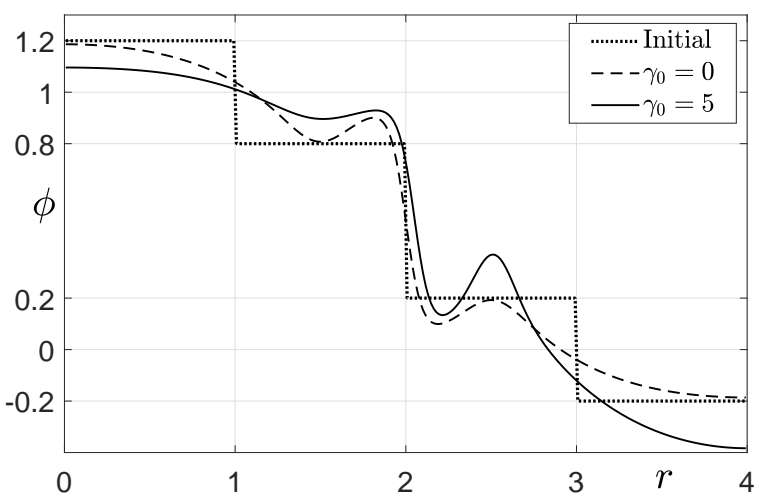

Figure 3: Computational results with $\gamma_{0}=0$ and $\gamma_{0}=5$ at $t=50 \Delta t$.

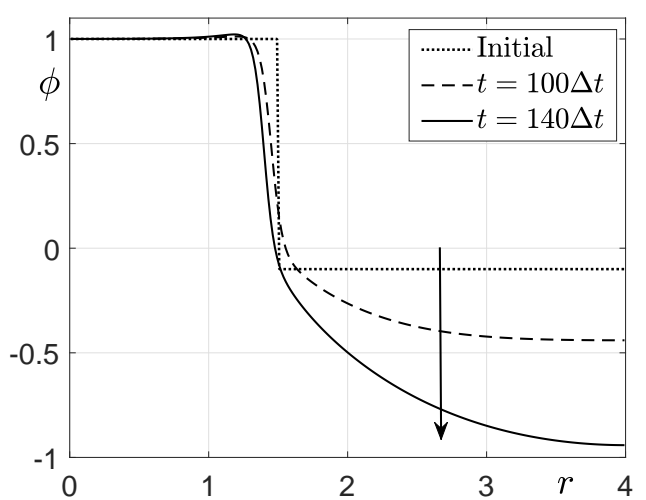

(a)

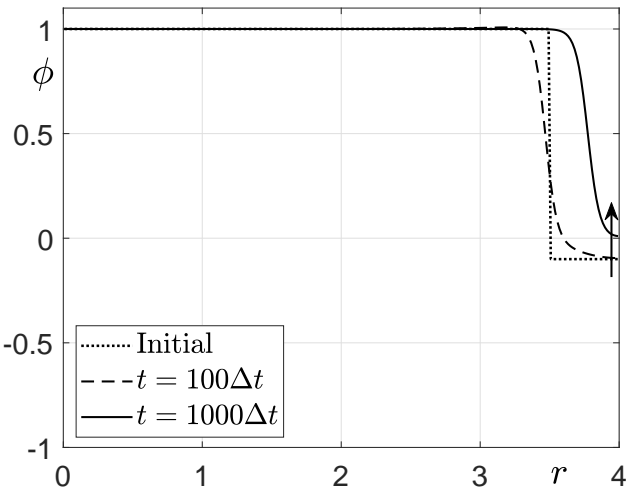

(b)

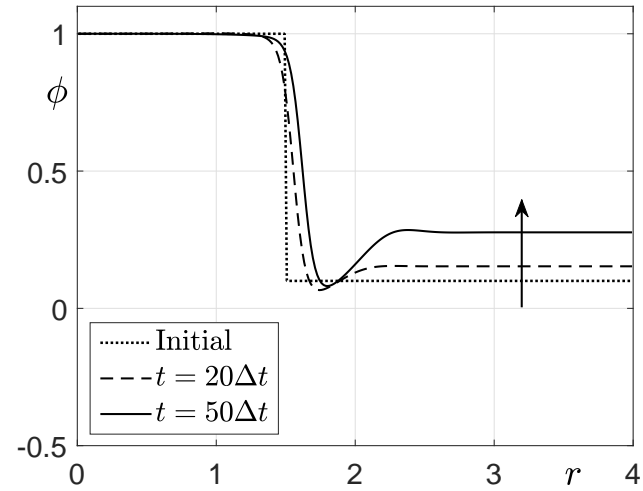

(c)

Figure 4: Comparison of computational results with different initial conditions: (a) blow-up, (b) stable, and (c) spurious growth. 


\subsection{Cut-off method for the logistic growth}

The cut-off method is to make all values of given function less than a critical value zero. To demonstrate that it is a simple and effective tool for use in the numerical computation, we perform numerical tests for the $\mathrm{CH}$ equation with the logistic source term using the cut-off in one-, two-, and three-dimensional spaces. Let us set an initial condition

$$
\phi(r, 0)=\frac{1}{2}\left[1-\tanh \left(\frac{r-r_{0}}{2 \sqrt{2} \epsilon}\right)\right],
$$

where $r_{0}$ is an interface position. Then, we have the following closed-form solution for Eqs. (2.1) and (2.2):

$$
\phi(r, t)=\frac{1}{2}\left[1-\tanh \left(\frac{r-r_{0}-\sqrt{2} \gamma_{0} \epsilon t}{2 \sqrt{2} \epsilon}\right)\right] .
$$

First, we solve Eqs. (2.6) and (2.7) numerically in one-dimensional space, i.e., $d=$ 1. Figs. 5(a) and 5(b) show the snapshots of the analytic and numerical solutions on $\Omega=(0,3)$ with $\gamma_{0}=0.5$ and $\gamma_{0}=5$, respectively. Here, $r_{0}=1, N_{r}=256, h=\frac{3}{N_{r}}$, and $\epsilon=\epsilon_{16}$ are used. The temporal step size is $\Delta t=0.005$, and the final times are (a) $T=5000 \Delta t$ and (b) $T=500 \Delta t$. If $\gamma_{0}$ is very small, then we have a good solution until $t=5000 \Delta t$ as shown in Fig. 5(a). However, if $\gamma_{0}$ is large, then Fig. 5(b) shows a big difference between the numerical solution and the analytic solution at $t=500 \Delta t$ because of the spurious growth, that is, the solution is unstable.

To resolve this problem, we propose the cut-off logistic source term

$$
\gamma_{\mathrm{c}}(\phi)= \begin{cases}\gamma_{0} \phi(1-\phi), & \text { if } \phi>\phi^{*}, \\ 0, & \text { otherwise, }\end{cases}
$$

where $\phi^{*}$ is a critical value. Note that the cut-off source term should have an important role in avoiding both the blow-up and the spurious growth of the numerical solutions.

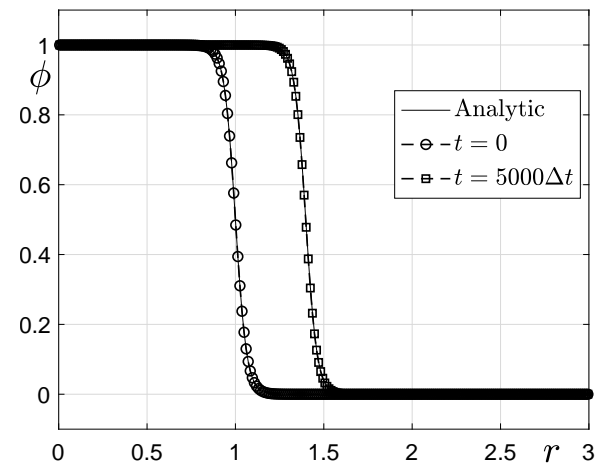

(a)

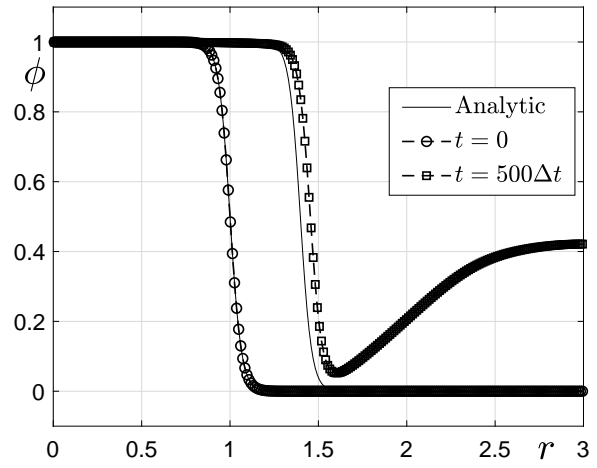

(b)

Figure 5: (a) and (b) are snapshots of the analytic and numerical solutions with $\gamma_{0}=0.5$ and $\gamma_{0}=5$ in the symmetric space, respectively. 


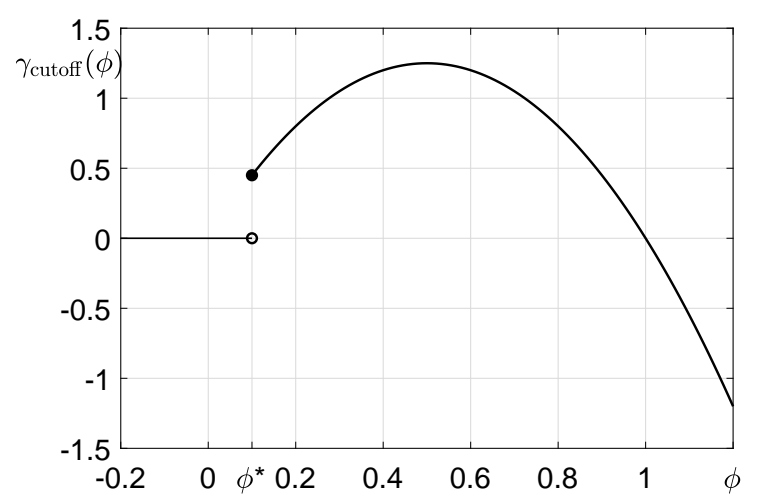

Figure 6: Schematic of the cut-off logistic source term $\gamma_{\mathrm{c}}(\phi)$ with $\gamma_{0}=1$ and $\phi^{*}=0.1$.

Thus, we choose a small positive value, $\phi^{*}=0.1$. Fig. 6 shows a schematic of the cut-off logistic source term, $\gamma_{\mathrm{c}}(\phi)$ with $\gamma_{0}=1$ and $\phi^{*}=0.1$.

Figs. 7(a) and 7(b) show snapshots of the analytic and numerical solutions with (a) $\phi^{*}=0.5$ and (b) $\phi^{*}=0.001$, respectively, in the symmetric space. Here, the parameters used are $\gamma_{0}=5, r_{0}=1, N_{r}=256, h=\frac{3}{N_{r}}, \Delta t=0.005, T=500 \Delta t$, and $\epsilon=\epsilon_{16}$. We observe that small concentration does not grow and overall growth is slower than what is expected if the critical value is too large.

Next, we consider Eqs. (2.6) and (2.7) with the logistic growth source term $\gamma(\phi)=$ $\gamma_{0} \phi(1-\phi)$ in two-dimensional space, i.e., $d=2$. As the radius $r$ increases, the curvature becomes smaller. Therefore, using the initial condition (3.6) with large $r_{0}$, we can compare the analytic solution and the numerical solutions (3.7) in two-dimensional space.

We perform the numerical tests without applying the cut-off as shown in Fig. 8. Figs. 8(a) and 8(b) show the snapshots of the analytic and numerical solutions on $\Omega=(100,103)$ with $\gamma_{0}=0.5$ and $\gamma_{0}=5$, respectively. Here, $r_{0}=101, N_{r}=256$,

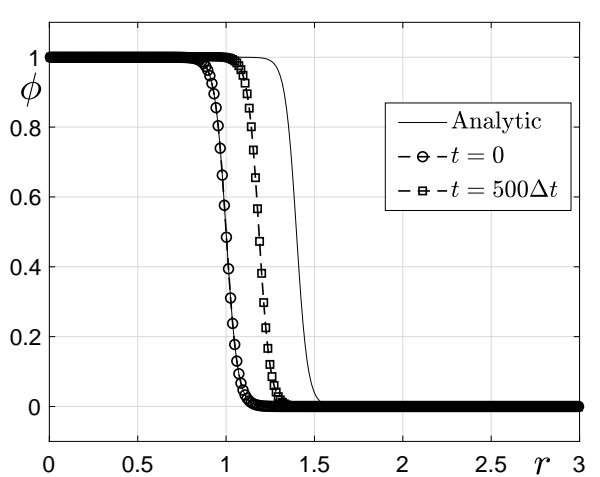

(a)

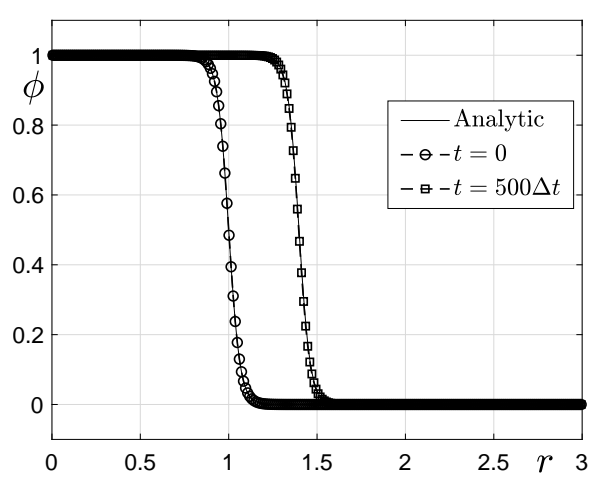

(b)

Figure 7: Snapshots of the analytic and numerical solutions with $\gamma_{0}=5$ in the symmetric space. (a) $\phi^{*}=0.5$ and (b) $\phi^{*}=0.001$. 


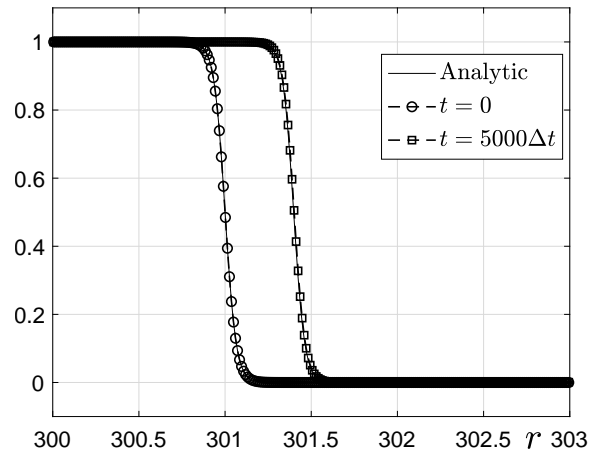

(a)

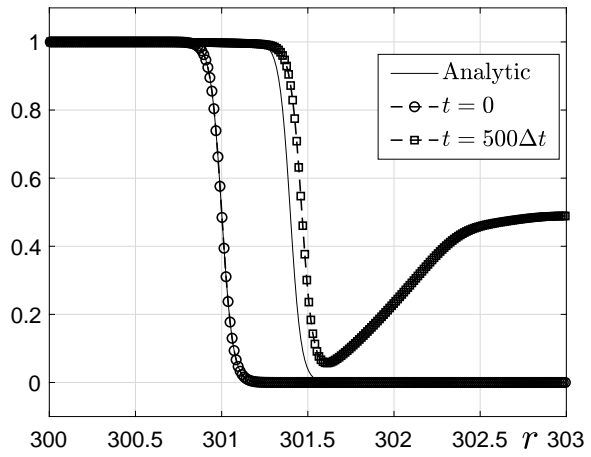

(b)

Figure 8: (a) and (b) are snapshots of the analytic and numerical solutions with $\gamma_{0}=0.5$ and $\gamma_{0}=5$ in the radially symmetric space, respectively.

$h=\frac{3}{N_{r}}$, and $\epsilon=\epsilon_{16}$ are used. The temporal step size is $\Delta t=0.005$ and the final times are (a) $T=5000 \Delta t$ and (b) $T=500 \Delta t$. With small $\gamma_{0}$, a good result is illustrated in Fig. 8(a), while large $\gamma_{0}$ makes the numerical solution unstable in Fig. 8(b).

When applying the cut-off, Figs. 9(a) and 9(b) show snapshots of the analytic and numerical solutions with $\phi^{*}=0.5$ and $\phi^{*}=0.001$, respectively, in the radially symmetric space. Here, the parameters used are $r_{0}=101, N_{r}=256, h=\frac{3}{N_{r}}, \gamma_{0}=0.5$, $\Delta t=0.005, T=500 \Delta t$, and $\epsilon=\epsilon_{16}$. With large $\phi^{*}$, the growth of the numerical solution is slower than that of the analytic solution because it does not grow well in small concentrations.

Finally, we consider Eqs. (2.6) and (2.7) with the initial condition (3.6) in threedimensional space, i.e., $d=3$. Figs. 10(a) and 10(b) show the snapshots of the analytic and numerical solutions on $\Omega=(100,103)$ with $\gamma_{0}=0.5$ and $\gamma_{0}=5$, respectively. Here, $r_{0}=101, N_{r}=256, h=\frac{3}{N_{r}}$, and $\epsilon=\epsilon_{16}$ are used. The temporal step size is $\Delta t=0.005$ and the final times are (a) $T=5000 \Delta t$ and (b) $T=500 \Delta t$. Because of large $\gamma_{0}$, the

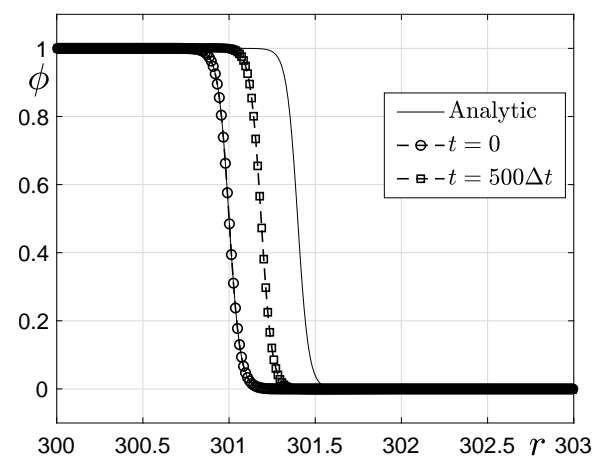

(a)

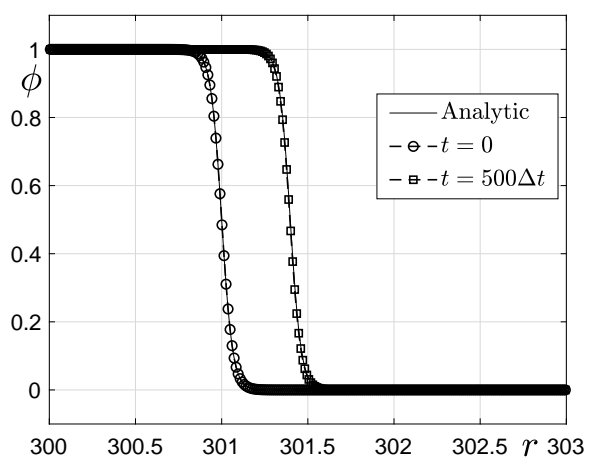

(b)

Figure 9: Snapshots of the analytic and numerical solutions with $\gamma_{0}=0.5$ in the radially symmetric space. (a) $\phi^{*}=0.5$ and (b) $\phi^{*}=0.001$. 


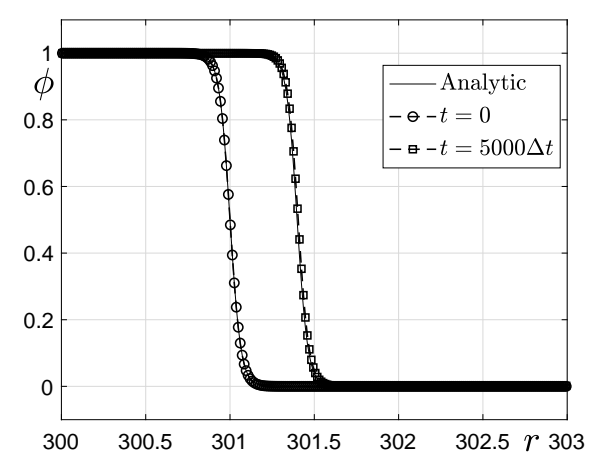

(a)

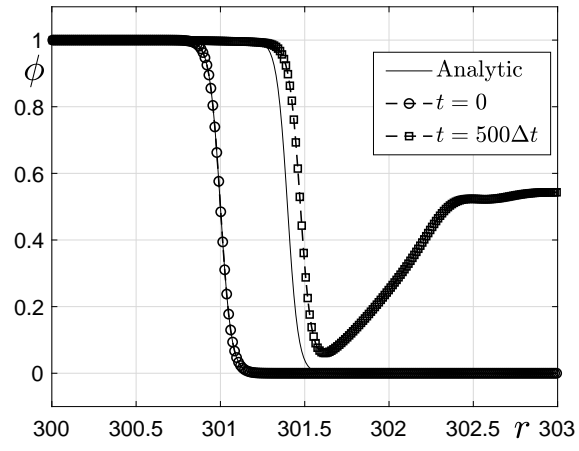

(b)

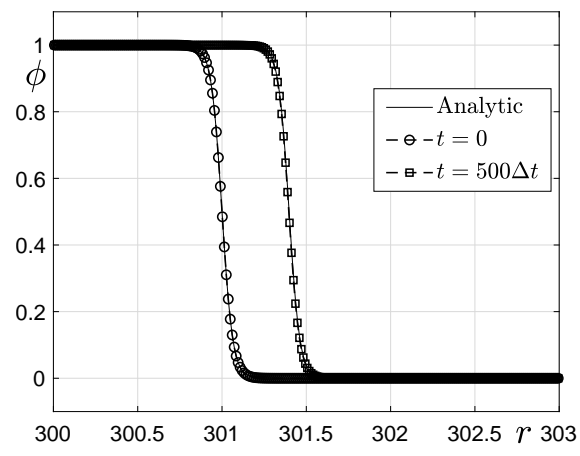

(c)

Figure 10: (a) and (b) are snapshots of the analytic and numerical solutions with $\gamma_{0}=0.5$ and $\gamma_{0}=5$ in the spherically symmetric space, respectively. (c) is the result with $\gamma_{0}=0.5$ and the cut-off source term with $\phi^{*}=0.001$ is used.

numerical solution is unstable as shown in Fig. 10(b). Fig. 10(c) shows the snapshot of the analytic and numerical solutions with the cut-off logistic source term. Here, we use $\gamma_{0}=0.5$ and $\phi^{*}=0.001$ and obtain an appropriate result numerically compared with the analytic solution.

In this section, we have so far conducted numerical tests to investigate the effects of the cut-off method when solving the $\mathrm{CH}$ equation with the logistic source term. Without the cut-off, the solution of the $\mathrm{CH}$ equation with the logistic source term can be unstable, therefore, we adopt the cut-off method to avoid the situation. However, it is difficult to choose the proper cut-off parameter $\phi^{*}$ because there is no theoretical criterion for selecting the value. Furthermore, there is a wide range of $\phi^{*}$ values suitable for the desired result, which varies considerably depending on the initial conditions or the set of parameters used. Thus, we perform a numerical experiment to find appropriate values of $\phi^{*}$ which makes the numerical solutions stable according to $\gamma_{0}$. Here, the same initial condition and parameters used in the previous three-dimensional simulation are taken, except for $\gamma_{0}$ and $\phi^{*}$. Fig. 11 illustrates the pairs of $\gamma_{0}$ and $\phi^{*}$. The unstable and stable solutions are represented by stars and open circles, respectively. To get stable solutions, we have to use the cut-off parameter $\phi^{*}$ depending on the value of $\gamma_{0}$ as shown in Fig. 11. 


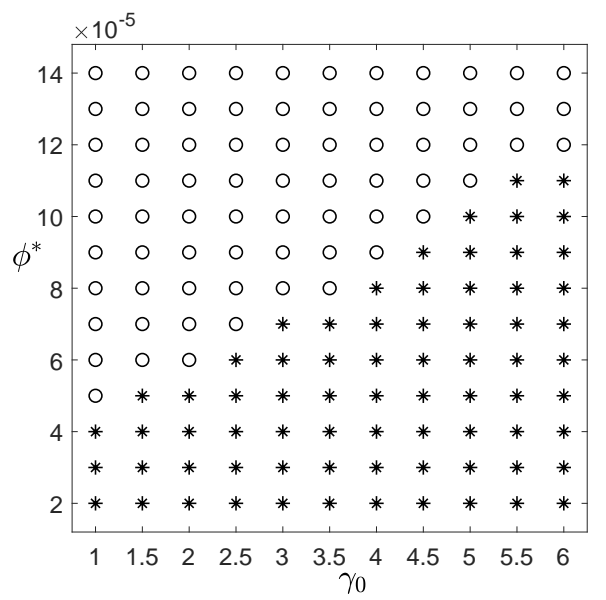

Figure 11: Stars and open circles indicate that numerical solutions are unstable and stable, respectively, with the pair of $\gamma_{0}$ and $\phi^{*}$.

\subsection{D Tumor growth simulation}

Through the benchmark tests, we verify that the instability of numerical solutions can be handled with the proper cut-off. Now, we demonstrate tumor growth combined with cut-off logistic growth term and death term. We modify the source term using the governing equation in [2] and delta function in [22]:

$$
\gamma(\phi)=\frac{\lambda_{g}}{\sqrt{2} \epsilon} \phi(1-\phi)-\lambda_{d} \phi
$$

where $\lambda_{g}$ and $\lambda_{d}$ are the growth and death coefficients. Here, $\frac{\phi(1-\phi)}{\sqrt{2} \epsilon}$ can replace $\frac{3 \sqrt{2}}{\epsilon} \phi^{2}(1-\phi)^{2}$ which is a smoothed dirac delta function because of the factor $\frac{3 \sqrt{2}}{\epsilon}$ [22]. We solve the $\mathrm{CH}$ equation (2.10) with a source term (3.9). The boundary condition is the homogeneous Neumann boundary condition (2.13). To match scaling parameter values as in [2], we take $\lambda_{g}=\frac{560 \epsilon_{0}}{3 \sqrt{2}}, \lambda_{d}=11.5$, and $\epsilon_{0}=\epsilon=0.00625$. The spatial step size $h=0.009375, N=N_{x}=N_{y}=256$, and the temporal step size $\Delta t=0.0032$ are used. The initial condition in the computational domain $\Omega=(0,2.4) \times(0,2.4)$ is

$$
\phi(x, y, 0)=\frac{1}{2}\left[1-\tanh \left(\frac{1}{2 \sqrt{2} \epsilon}\left(\sqrt{2(x-1.2)^{2}+0.25(y-1.2)^{2}}-0.1\right)\right)\right] .
$$

Fig. 12(a) shows snapshots at $t=0,25 \Delta t, 40 \Delta t$. Without the cut-off, the numerical solution is unstable. Therefore, it is need to use the cut-off source term introduced in Section 3.2. We consider the cut-off logistic source term

$$
\gamma_{\mathrm{c}}(\phi)= \begin{cases}\frac{\lambda_{g}}{\sqrt{2} \epsilon} \phi(1-\phi)-\lambda_{d} \phi, & \text { if } \phi>\phi^{*}, \\ 0, & \text { otherwise. }\end{cases}
$$


(a)
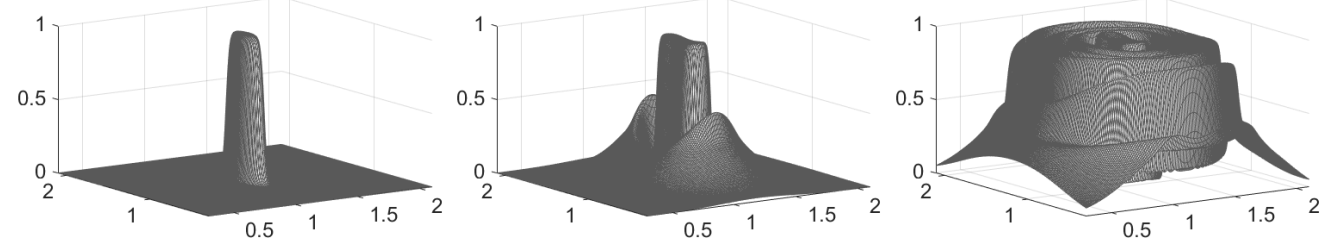

(b)
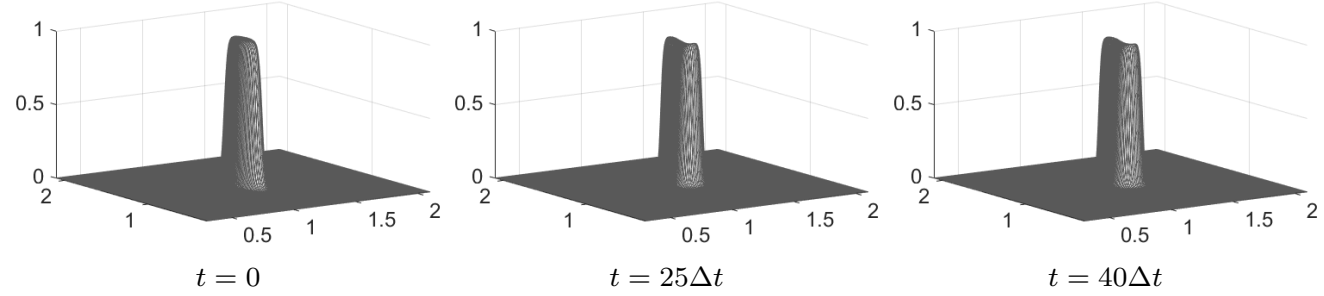

Figure 12: (a) and (b) are snapshots at $t=0,25 \Delta t, 40 \Delta t$, respectively. Without the cut-off, the numerical solution is unstable as shown in (a).

Here, we take $\phi^{*}=0.07$. In contrast to Fig. 12(a), when we adopt the cut-off logistic source term, the numerical solution is stable as shown in Fig. 12(b).

Fig. 13 shows the tumor growth results at $t=0,1000 \Delta t, 2000 \Delta t, 3000 \Delta t$. By using the cut-off logistic source term, the numerical solution becomes stable.

\subsection{D Tumor growth simulation}

Next, we carry out numerical tests in three-dimensional space using the $\mathrm{CH}$ equation (2.14) and the source term (3.9). The boundary condition is the homogeneous Neumann boundary condition (2.17). The initial condition in the computational domain $\Omega=(0,1.65) \times(0,1.65) \times(0,1.65)$ is

$$
\begin{aligned}
& \phi(x, y, z, 0) \\
= & \frac{1}{2}\left[1-\tanh \left(\frac{1}{2 \sqrt{2} \epsilon}\left(\sqrt{2(x-0.825)^{2}+0.5(y-0.825)^{2}+0.25(z-0.825)^{2}}-0.1\right)\right)\right]
\end{aligned}
$$

We use the parameters: $N_{x}=N_{y}=N_{z}=128, h=\frac{1.65}{128}, \Delta t=0.0032$, and $\epsilon_{0}=\epsilon=$ $0.00625, \lambda_{g}=\frac{1008 \epsilon_{0}}{3 \sqrt{2}}$, and $\lambda_{d}=29.9$

Figs. 14(a)-14(c) are temporal evolutions without the cut-off at $t=0,15 \Delta t, 25 \Delta t$, respectively. The first three columns from left to right represent the isosurfaces at $\phi=0.2,0.5,0.8$ and the last column illustrates the snapshots in cross section parallel to $x z$-plane at $y=0.825$. The numerical simulation without the cut-off demonstrates that the numerical solution is unstable as shown in Fig. 14.

Let us consider the cut-off logistic source term (3.11) for logistic growth and take $\phi^{*}=0.07$. Figs. 15(a)-15(d) present temporal evolutions of the tumor growth simulation at $t=0,500 \Delta t, 800 \Delta t, 1200 \Delta t$, respectively. The isosurfaces and snapshots in 


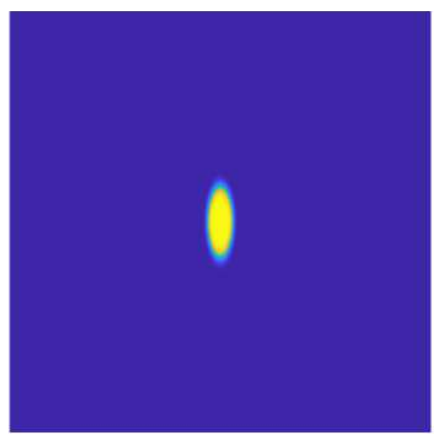

(a)

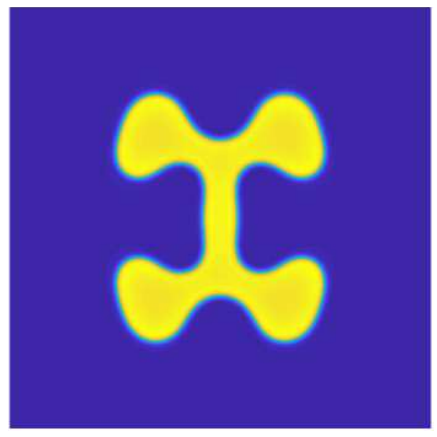

(c)

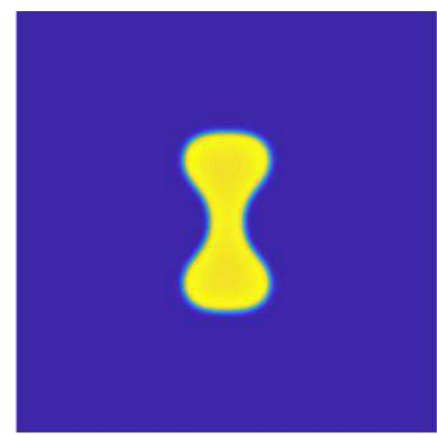

(b)

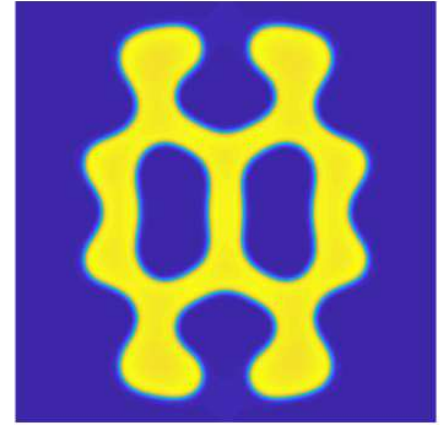

(d)

Figure 13: Temporal evolutions with the cut-off source term (3.11) and $\phi^{*}=0.07$. (a), (b), (c), and (d) are snapshots at $t=0,1000 \Delta t, 2000 \Delta t, 3000 \Delta t$, respectively.

(a)

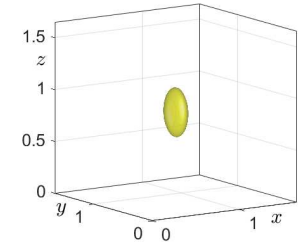

(b)

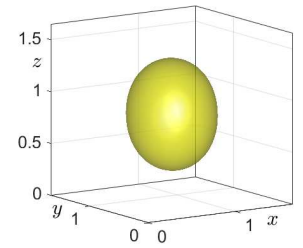

(c)

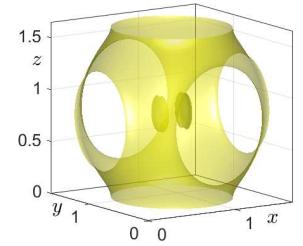

$\phi=0.2$
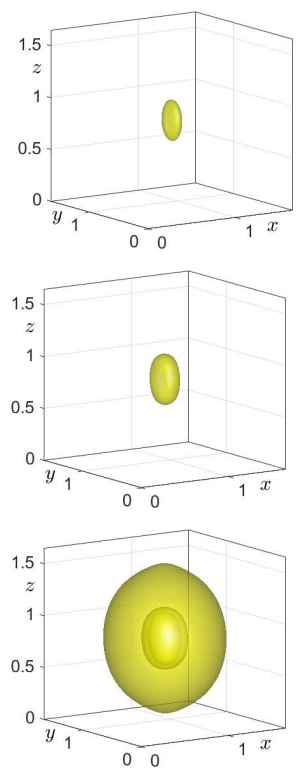

$\phi=0.5$
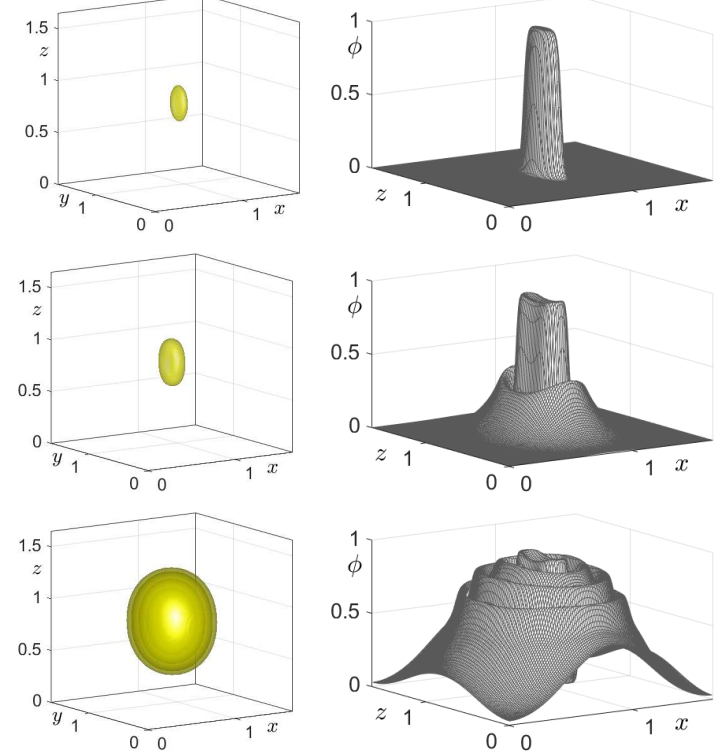

$\phi=0.8$

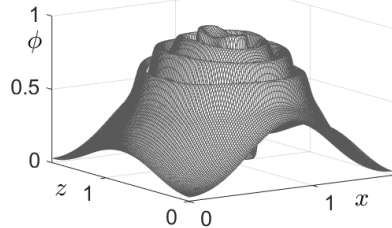

$y=0.825$

Figure 14: Isosurfaces at $\phi=0.2,0.5,0.8$ and snapshots in cross section parallel to $x z$-plane at $y=0.825$ without the cut-off. (a), (b), and (c) are temporal evolutions at $t=0,15 \Delta t, 25 \Delta t$, respectively. 

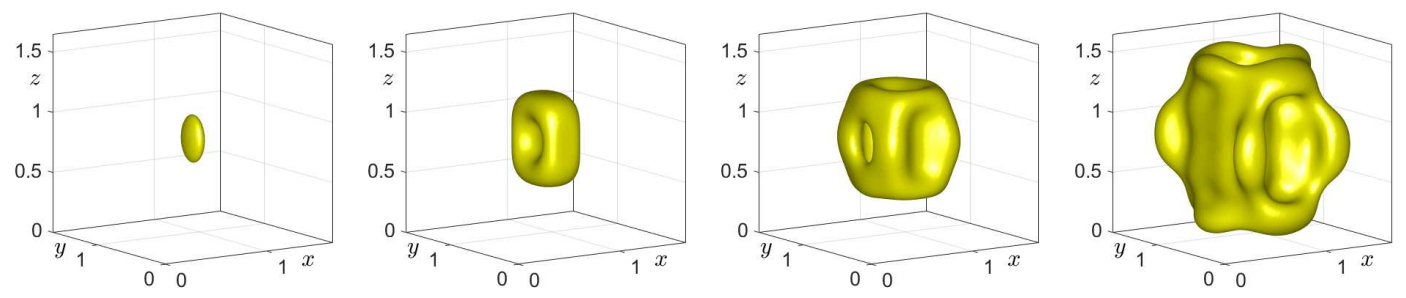

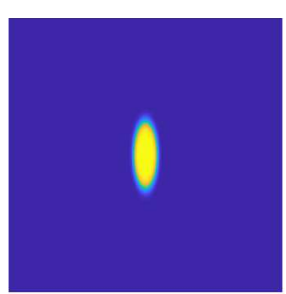

(a)

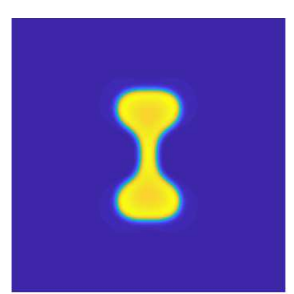

(b)

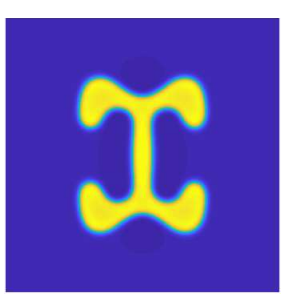

(c)

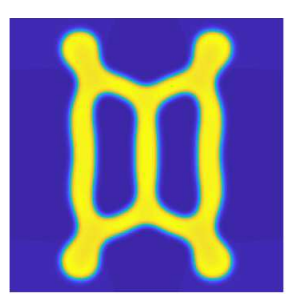

(d)

Figure 15: With the cut-off logistic source term (3.9) and $\phi^{*}=0.07,(a),(b),(c)$, and (d) are isosurfaces at $\phi=0.5$ (top) and snapshots in cross section parallel to $x z$-plane (bottom) at $t=0,500 \Delta t, 800 \Delta t, 1200 \Delta t$, respectively.

cross section of the isosurfaces parallel to $x z$-plane at $y=0.825$ are presented on the top and bottom in Fig. 15.

\section{Conclusions}

We considered the effect of source terms on the evolutionary dynamics of the $\mathrm{CH}$ equation. First, we observed the evolution of solution profiles for the logistic growth source term and presented a cut-off logistic source term. We used a benchmark problem and the nonlinear multigrid method for the $\mathrm{CH}$ equation. Then, we considered the source term for tumor growth simulation which is represented by smoothed dirac delta functions and carried out numerical experiments in two- and three-dimensional space. Applying the proposed cut-off logistic source term, we can simulate the tumor growth simulation. In a future work, we plan to deal with the effect of source terms of the Allen-Cahn type equation like a mathematical model of tumor angiogenesis in [33].

\section{Acknowledgments}

The first author (C. Lee) was supported by Basic Science Research Program through the National Research Foundation of Korea (NRF) funded by the Ministry of Education (NRF-2019R1A6A3A13094308). The corresponding author (J.S. Kim) was supported by Basic Science Research Program through the National Research Foundation of Korea (NRF) funded by the Ministry of Education (NRF-2019R1A2C1003053). The authors thank the editor and the reviewers for their constructive and helpful comments on the revision of this article. 


\section{References}

[1] M. A. Alias And P. R. Buenzli, A level-set method for the evolution of cells and tissue during curvature-controlled growth, Int. J. Numer. Method. Biomed. Eng., 36(1) (2020), e3279.

[2] A. C. Aristotelous, O. A. Karakashian, and S. M. Wise, Adaptive, second order in time, primitive-variable discontinuous Galerkin schemes for a Cahn-Hilliard equation with a mass source, IMA J. Numer. Anal., 35(3) (2015), 1167-1198.

[3] A. L. Bertozzi, S. Esedoglu, AND A. Gillette, Inpainting of binary images using the Cahn-Hilliard equation, IEEE Trans. Image Process., 16(1) (2006), 285-291.

[4] W. E. BOYCE AND R. C. DiPrima, Elementary differential equations and boundary value problems, Wiley, 2008.

[5] J. W. CAHn AND J. E. Hilliard, Free energy of a non-uniform system I. Interfacial free energy, J. Chem. Phys., 28(2) (1958), 258-267.

[6] L. Cherfils, A. Miranville, AND S. Zelik, On a generalized Cahn-Hilliard equation with biological applications, Discrete Contin. Dyn. B, 19(7) (2014), 2013-2026.

[7] D. S. Cohen AND J. D. MurraY, A generalized diffusion model for growth and dispersal in a population, J. Math. Biol., 12 (1981), 237-249.

[8] V. CRistini, X. Li, J. S. Lowengrub, AND S. M. Wise, Nonlinear simulations of solid tumor growth using a mixture model: invasion and branching, J. Math. Biol., 58(4-5) (2009), 723.

[9] D. J. EYRE, An unconditionally stable one-step scheme for gradient systems, Preprint (1998), 1-15. http://www.math.utah.edu/ eyre/research/methods/stable.ps.

[10] H. FAKIH, A Cahn-Hilliard equation with a proliferation term for biological and chemical applications, Asymptotic Analysis, 94(1-2) (2015), 71-104.

[11] H. B. Frieboes, F. Jin, Y. L. Chuang, S. M. Wise, J. S. Lowengrub, AND V. CRistini, Three-dimensional multispecies nonlinear tumor growth-II: tumor invasion and angiogenesis, J. Theor. Biol., 264(4) (2010), 1254-1278.

[12] A. Hawkins-DaARud, K. G. van der Zee, and J. Tinsley Oden, Numerical simulation of a thermodynamically consistent four-species tumor growth model, Int. J. Numer. Meth. Bio. Eng., 28(1) (2012), 3-24.

[13] Q. HuANG, D. LI, AND J. ZHANG, Numerical investigations of a class of biological models on unbounded domain, Numer. Math. Theor. Meth. Appl., 12(1) (2019), 169-186.

[14] D. JEONG, Y. CHOI, AND J. KIM, A benchmark problem for the two- and three-dimensional Cahn-Hilliard equations, Commun. Nonlinear Sci. Numer. Simul., 61 (2018), 149-159.

[15] D. JEONG AND J. Kim, Phase-field model and its splitting numerical scheme for tissue growth, Appl. Numer. Math., 117 (2017), 22-35.

[16] J. JIANG, K. GARIKIPATI, AND S. RUDRARAJU, A diffuse interface framework for modelling the evolution of multi-cell aggregates as a soft packing problem due to growth and division of cells, Bull. Math. Biol., 81(8) (2019), 3282-3300.

[17] E. KhAin AND L. M. SANDER, Generalized Cahn-Hilliard equation for biological applications, Phys. Rev. E, 77(5) (2008), 051129.

[18] J. KIM, A numerical method for the Cahn-Hilliard equation with a variable mobility, Commun. Nonlinear Sci. Numer. Simul., 12 (2007), 1560-1571.

[19] J. KIM, Phase-field models for multi-component fluid flows, Commun. Comput. Phys., 12(3) (2012), 613-661.

[20] I. KLAPPER AND J. DOCKeRY, Role of cohesion in the material description of biofilms, Phys. Rev. E., 74 (2006), 031902. 
[21] D. Lee, J. Y. Huh, D. Jeong, J. Shin, A. Yun, And J. Kim, Physical, mathematical, and numerical derivations for the Cahn-Hilliard equations, Comput. Mater. Sci., 81 (2014), 216-225.

[22] H. G. LeE And J. Kim, Regularized Dirac delta functions for phase field models, Int. J. Numer. Methods Eng., 91(3) (2012), 269-288.

[23] H. G. LEE, J. YANG, J. PARK, AND J. Kim, Effect of space dimensions on equilibrium solutions of Cahn-Hilliard and conservative Allen-Cahn equations, Numer. Math. Theor. Meth. Appl., 13(3) (2020).

[24] C. LU, W. HUANG, AND E. S. VAN VLECK, The cutoff method for the numerical computation of nonnegative solutions of parabolic PDEs with application to anisotropic diffusion and lubrication-type equations, J. Comput. Phys., 242 (2013), 24-36.

[25] V. Mohammadi And M. Dehghan, Simulation of the phase field Cahn-Hilliard and tumor growth models via a numerical scheme: Element-free Galerkin method, Comput. Meth. Appl. Mech. Eng., 345 (2019), 919-950.

[26] J. D. MURRAY, Mathematical Biology I: An Introduction, Interdisciplinary Applied Mathematics, Mathematical Biology, Springer, 2002.

[27] B. NicolaenKo, B. ScheureR, AND R. Temam, Some global dynamical properties of a class of pattern formation equations, Commun. Part. Diff. Eq., 14(2) (1989), 245-297.

[28] A. Oron, S. H. Davis, AND S. G. BANKOFF, Long-scale evolution of thin liquid films, Rev. Mod. Phys., 69 (1997), 931-980.

[29] S. Sanga, H. B. Frieboes, X. Zheng, R. Gatenby, E. L. Bearer, and V. Cristini, Predictive oncology: a review of multidisciplinary, multiscale in silico modeling linking phenotype, morphology and growth, Neuroimage, 37 (2007), S120-S134.

[30] J. SHEN AND X. YANG, Energy stable schemes for Cahn-Hilliard phase-field model of twophase incompressible flows, Chinese Ann. Math., Ser. B, 31(5) (2010), 743-758.

[31] J. SHIN, H. G. LEE, AND J. Y. LEe, First and second order numerical methods based on a new convex splitting for phase-field crystal equation, J. Comput. Phys., 327 (2016), 519542.

[32] U. Trottenberg, C. Oosterlee, And A. Schüller, Multigrid, Academic Press, 2001.

[33] G. Vilanova, I. Colominas, And H. Gomez, A mathematical model of tumour angiogenesis: growth, regression and regrowth, J. R. Soc. Interface, 14(126) (2017), 20160918.

[34] S. M. Wise, J. S. Lowengrub, H. B. Frieboes, And V. Cristini, Three-dimensional multispecies nonlinear tumor growth-I: model and numerical method, J. Theor. Biol., 253(3) (2008), 524-543.

[35] X. WU, G. J. van ZWIETEN, AND K. G. VAN DER ZEe, Stabilized second-order convex splitting schemes for Cahn-Hilliard models with application to diffuse-interface tumor-growth models, Int. J. Numer. Method. Biomed. Eng., 30(2) (2014), 180-203.

[36] X. YANG, Numerical approximations for the Cahn-Hilliard phase field model of the binary fluid-surfactant system, J. Sci. Comput., 74(3) (2018), 1533-1553. 